\title{
Uso de objetos de aprendizagem no ensino da Lei dos Senos e da Lei dos Cossenos
}

Resumo: Atualmente, o ensino de Matemática se apresenta em diversos contextos. A Trigonometria, por exemplo, pode ser lecionada de forma interdisciplinar e dinâmica. Neste sentido, é proposto investigar o ensino de Trigonometria, dando ênfase a Lei dos Senos e a Lei dos Cossenos, utilizando, para isso, objetos de aprendizagem. Nessa investigação, é realizada uma Sequência de Ensino Investigativa, auxiliada por um simulador da Plataforma PhET de modo a integrar os conteúdos de Trigonometria com os de Vetores. Os resultados da Atividade Avaliativa e do Questionário de Autoavaliação apontam que houve interação entre os estudantes durante a aula, bem como a apropriação de conceitos, ocorrendo assim os processos de ensino e de aprendizagem, de maneira a mostrar que existe relação entre a Trigonometria e Vetores. Assim, podese concluir que, a Plataforma PhET é uma alternativa didático-metodológica para se ensinar Trigonometria, a partir do estudo de Vetores.

Palavras-chave: Plataforma PhET. Vetores. Sequência de Ensino Investigativa.

\section{Patricia de Souza Moura}

Mestranda em Ensino pela

Universidade do Estado do Rio

Grande do Norte (UERN), campus

Pau dos Ferros. Rio Grande do Norte,

Brasil..

(iD) orcid.org/0000-0002-9069-8320

$\bowtie$ patryciacedro@gmail.com

Otávio Paulino Lavor

Doutor em Engenharia Elétrica.

Professor do Programa de Pós-

Graduação em Ensino da

Universidade Federal Rural do Semi-

Árido (UFERSA), campus Pau dos

Ferros. Rio Grande do Norte, Brasil.

(iD) orcid.org/0000-0001-5237-3392

$\bowtie$ otavio.lavor@ufersa.edu.br

Recebido em 14/01/2021 Aceito em 17/03/2021

Publicado em 10/04/2021

\section{Use of learning objects in the teaching of the Senos Law and the Cosines Law}

Abstract: The teaching of Mathematics is presented in several contexts today, such as Trigonometry, which can be taught in an interdisciplinary and dynamic way. In this sense, it is proposed to investigate the teaching of Trigonometry, particularly with the Law of Sines and Cosines, using learning objects. In this investigation, an Investigative Teaching Sequence will be carried out, aided by a simulator of the PhET platform in order to integrate the contents of Trigonometry and Vectors. The results of the evaluative activity and the satisfaction requirement indicate that there was interaction between the students during the class, as well as satisfactory concepts, occurring the teaching and learning process, also showing that there is a relationship between Trigonometry and Vectors. Thus, it can be concluded that a PhET platform is an alternative to using Trigonometry, using Vectors.

Keywords: PhET Platform. Vectors. Investigative Teaching Sequence.

\section{Uso de objetos de aprendizaje en la enseñanza de la Ley de Senos y la Ley de Cosenos}

Resumen: La enseñanza de las Matemáticas se presenta hoy en varios contextos, como el de la Trigonometría, que se puede enseñar de forma interdisciplinar y dinámica. En 
este sentido, se propone investigar la enseñanza de la Trigonometría, particularmente con la Ley de Senos y Cosenos, utilizando objetos de aprendizaje. En esta investigación se llevará a cabo una Secuencia de Enseñanza Investigativa, con la ayuda de un simulador de plataforma PhET para integrar los contenidos de Trigonometría y Vectores. Los resultados de la actividad evaluativa y el cuestionario de satisfacción indican que hubo interacción entre los estudiantes durante la clase, así como conceptos satisfactorios, ocurriendo los procesos de enseñanza y de aprendizaje, mostrando también que existe una relación entre Trigonometría y Vectores. Así, se puede concluir que la plataforma PhET es una alternativa a la enseñanza de la Trigonometría mediante Vectores.

Palabras clave: Plataforma PhET. Vectores. Secuencia de Enseñanza Investigativa.

\section{Introdução}

$\mathrm{O}$ ensino de Matemática quando aliado às tecnologias da informação e comunicação (TIC) pode contribuir para os processos de ensino e de aprendizagem. Assim, ao ser realizado um planejamento que possa flexibilizar a exposição do conteúdo, trazendo a aplicação tecnológica como um recurso expositivo, desenvolvendo exemplos práticos que levem o estudante a compreender o conteúdo — não apenas de forma teórica, mas também na prática - e desenvolvendo habilidades, torna efetivo o referido processo.

Aprender Matemática requer do estudante atenção e entusiasmo, assim como acontece nas demais áreas do conhecimento. É preciso concebê-la dessa forma, mesmo que exista diversos comentários a respeito da visão de muitos estudantes sobre a Matemática como uma disciplina difícil de compreensão. Essa é uma concepção que passa a ser entendida como um estereotipo, criada pela sociedade desde quando a Matemática era apenas ensinada como mecanização e treino, a partir de longas listas de exercícios, como mesmo nível de raciocínio, na qual o único objetivo era transmissão e acúmulo de uma gama de informações, geralmente, descontextualizadas.

Assim, a dificuldade contrasta com a facilidade, pois tudo aquilo que é dominado consequentemente se torna mais fácil de realizar; dessa forma, a dificuldade está relacionada a algo que ainda não é dominado; um obstáculo que, ao ser vencido, pode ser eliminado ou minimizado, ou seja, envolvendo questões de superação pessoal. (MASOLA e ALLEVATO, 2019).

A longo dos tempos, o ensino da Matemática veio sofrendo muitas modificações, levando em consideração que há um tempo, a Matemática era lecionada de forma mecânica, desvinculada de contextos e significados, desprovida dos porquês e, tendo 
como objetivo, a memorização de fórmulas e o "treino de contas". Assim, oportunizar o/a aluno/a, a se envolver com as aplicações da Matemática: a oportunidade de usar os conceitos matemáticos em seu dia - a - dia favorece uma atitude positiva do/a aluno/a em relação a Matemática. Desse modo, não basta saber mecanicamente as operações matemáticas, é preciso saber como e quando usar esses conceitos. (BUENO, ALENCAR e MILLONES, 2017).

O ensino não é apenas um processo de entrada e saída de informações, nem pode ser considerado a partir de uma área do desenvolvimento isolada e marcada pela certeza e pela inalterabilidade. $\mathrm{O}$ ensino é um processo educativo que deve estar sendo sempre avaliado e revisado, para que assim atenda às necessidades dos estudantes, de forma a alcançar uma compreensão de todo o contexto no qual este está inserido (PERIUS, 2012).

\begin{abstract}
Essas contribuições coletivas refletem também em como o ensino de matemática encontra-se presente em diversas disciplinas afins e não afinas, compreender sua aplicação em diversos contextos e demonstrar isso através do ensino, torna-se de fundamental importância, determina que uma ciência não necessariamente deve andar sozinha, mas contribuir efetivamente paras as demais, e vice-versa. Demonstrar ao estudante essa relação, favorece para que o mesmo consiga entender que a Matemática não é uma ciência isolada. (MOURA, RAMOS e LAVOR, 2020, p. 576).
\end{abstract}

Nesse contexto, então, as tecnologias de ensino desenvolvem um papel fundamental quando aplicadas ao ensino de Trigonometria. Por se tratar de um conteúdo da Matemática pouco explorado de uma forma prática, desenvolver as habilidades nele inseridas, utilizando recursos tecnológicos, pode contribuir para o desempenho dos estudantes diante do desenvolvimento cognitivo, a partir de propostas que possam facilitar a compreensão e o entendimento dos conceitos.

O uso de recursos tecnológicos contribui para modificar a metodologia de ensino e aprendizagem fazendo com que os assuntos trabalhados nas disciplinas tenham mais sentido, em particular, nas aulas de Matemática, uma das mais temidas pelos estudantes. O uso de tais recursos possibilita o desenvolvimento do conhecimento de maneira divertida e interativa, contribuindo assim para a motivação dos estudantes, pois, disponibilizar atividades atrativas, constitui-se como um instrumento eficaz à aprendizagem e à resolução de problemas, por meio da interação com o saber (AFONSO e MALDONADO, 2020).

Borges (2020) foi um dos que utilizou na sua pesquisa de mestrado a Modelagem 
Matemática para trabalhar o ensino de Trigonometria. Enquanto Rodrigues (2020) utilizou de forma conjunta a Modelagem matemática da voz, Robótica e a Trigonometria para o desenvolvimento de atividades interativas. Já Machado (2020) fez uso da ferramenta tecnológica GeoGebra para trabalhar o ensino de Funções Trigonométricas.

Dessa forma, tendo em vista a utilização das tecnologias no ensino de Matemática, este artigo traz como objetivo a particularização dessas aplicações na Trigonometria. Compreender a relação de teoria e prática, entender a importância da demonstração desses recursos diante da aprendizagem, de forma aplicável, sendo possível ocorrer a visualização e a interação do conteúdo ministrado por meio de recursos tecnológicos, possibilita ao estudante a aquisição de novos conhecimentos e habilidades.

Nesse sentido, este artigo consiste em uma proposta interdisciplinar que utiliza uma Sequência de Ensino Investigativo (SEI) com o propósito de envolver o ensino de Matemática, com os conteúdos envolvidos na Lei dos Senos e na Lei dos Cossenos, de modo interdisciplinar com a Física, a partir do conteúdo de Vetores, isto é, utilizando o simulador de Adição de Vetores da Plataforma PhET como objeto de aprendizagem.

\section{Objetos de aprendizagem no ensino de Matemática}

Os objetos de aprendizagem no ensino de Matemática contribuem para o desenvolvimento da aprendizagem no que tange à sua utilização de forma teórica e, ainda, prática. Dessa forma, a utilização de recursos digitais nesse processo possibilita uma abordagem interdisciplinar, compreendendo um aspecto importante e marcante, levando o estudante à interação com o conteúdo abordado, o objeto de aprendizagem utilizado e a metodologia que compreende a aplicação. Araújo e Bracho (2020) afirmam que os objetos de aprendizagem apresentam vantagens que podem ajudar os estudantes a terem uma relação afetiva e dinâmica com a aprendizagem e seus saberes.

\footnotetext{
Essa autonomia dada ao discente através do uso das tecnologia compreende e contribui para um desenvolvimento cognitivo no seu processo de aprendizagem, pois o discente, pode entender, além da teoria o que a disciplina aborda. Quando a tecnologia é aliada ao ensino, e a interdisciplinaridade, pode favorecer no processo desse desenvolvimento, sendo possível, através dela, demonstrar a relação entre teoria e prática e até onde uma disciplina se relacionada a outra (MOURA, RAMOS e LAVOR, 2020, p. 578).
}

Fernanda e Reis (2019) acreditam que a inclusão de novos métodos, técnicas e 
tecnologias fazem parte do contexto educacional atual e permite a utilização de vários recursos pedagógicos, que podem ser aproveitados em diversas modalidades de ensino. Essas novas tecnologias podem trazer benefícios para os processos de ensino e de aprendizagem.

Oliveira (2020) considera ser possível verificar a importância do ensino dos saberes do conhecimento mediante a inclusão de recursos educacionais digitais facilitadores de aprendizagem, que buscam contribuir de forma contínua com o ambiente escolar e acadêmico e, ainda, proporcionar satisfatório desenvolvimento no ensino e aprendizagem destes saberes.

Aguiar e Flôres (2014) compreendem que o objeto de aprendizagem se apresenta como uma ferramenta vantajosa de aprendizagem e instrução, podendo ser utilizada para o ensino de diversos conteúdos e revisão de conceitos.

Desse modo, torna-se possível admitir que os objetos de aprendizagem vêm para contribuir de forma significativa nos processos de ensino e de aprendizagem, proporcionando uma aula mais interativa e levando o estudante a compreender o conteúdo exposto de forma clara, compreensível e aplicável.

Lavor e Martins (2020) afirmam que, na sala de aula, o professor encontra dificuldades quando a aula ocorre de maneira tradicional, sem que desperte o interesse e a motivação de seus estudantes. Assim, faz-se necessário haver questionamentos, reflexões e mudanças sobre atividades educativas que possam tornar interessante aos estudantes a partir do uso de novas metodologias e de objetos de aprendizagem, fazendo com que questionem e desenvolvam autonomia cognitiva, ou seja, capacidade de pensar e resolver questões por si mesmo.

\footnotetext{
Compreendemos a resolução de problemas como uma metodologia desafiadora, problematizada a e dinâmica no processo de ensino aprendizagem da Matemática, que oportuniza aos estudantes, com os novos conhecimentos matemáticos discutidos no trabalho grupal, nos espaço s formativos para resolver os problemas propostos pela mediadora da aula ou por eles criados, experimentar e dar sentido aos conhecimentos que já têm (OLIVEIRA et al. 2021, p. 12).
}

Para o ensino de Matemática compreende-se, diante do exposto, que os objetos de aprendizagem contribuem para um processo de ensino e de aprendizagem de forma significativa, à medida que é utilizado nas aulas; favorece para uma aula dinâmica e 
possibilita a compreensão do conteúdo por meio da aplicação e exposição.

Silva, Severiano e Urzêda (2020) retratam que nesses ambientes, a linguagem matemática é apresentada com diversas ferramentas de interatividade, proporcionando o estímulo ao interesse e à compreensão. Aplicativos que retratam o ensino de Matemática têm sido, ao longo dos últimos anos, amplamente desenvolvidos e disponibilizados na perspectiva de colaborar na minimização das dificuldades de aprendizagem.

Os objetos de aprendizagem são aplicados em diversos campos do ensino, assim como na Matemática e na Física. Oliveira, Porto e Alves (2018) aplicaram "memes" que são utilizados em redes sociais digitais como Objetos de Aprendizagem na Cibercultura.

Araújo e Bracho (2020) utilizaram simuladores com o software GeoGebra com objetos de aprendizagem voltados para o ensino de Física, enquanto Cruz e Monteiro (2019) aproveitaram requisitos para que ocorresse o desenvolvimento dos objetos de aprendizagem reutilizáveis em dispositivos móveis.

Observa-se, então, que nos contextos mencionados e, em tantos outros, o processo de planejamento se torna importante e necessário pois é com base nele que são traçados os objetivos a serem alcançados. Segundo Silva, Teixeira e Müller (2019), mesmo diante de algumas dificuldades que provocam o desinteresse no estudante para a aprendizagem de Matemática, o objeto de aprendizagem vem proporcionar um estudo diferenciado com objetivo que ocorra a motivação e o interesse dele a refletir, observar e interagir com Matemática, portanto, de modo que possa contribuir para a aprendizagem dos conteúdos.

\section{Metodologia}

A presente pesquisa, caracterizada de cunho quanti-qualitativo, foi realizada em uma escola de tempo integral com dez estudantes do Ensino Médio da rede pública, no dia 12 de outubro de 2020 .

As Sequencias de Ensino Investigativas (SEI) são propostas por Carvalho (2013, p. 7) como sendo

sequências de atividades (aulas) abrangendo um tópico do programa escolar em que cada uma das atividades é planejada, sob o ponto de vista do material e das interações didáticas, visando proporcionar aos alunos: condições de trazer seus conhecimentos prévios para iniciarem os novos, terem ideias 
próprias e poder discuti-las com seus colegas e com o professor passando do conhecimento espontâneo ao científico e tendo condições de entenderem conhecimentos já estruturados por gerações anteriores.

Como exemplos de Sequências de Ensino Investigativos (SEI) empregadas no contexto educacional, têm-se as apresentadas por Tadiello (2020), que utilizou a SEI para prática laboratoriais no ensino de Ciências. Já Santos (2020) analisou a utilização da SEI para a Química Forense, enquanto Melo, Santo e Araújo (2020) empregaram o uso de recursos digitais junto com a SEI para o ensino de soluções no Ensino Médio.

Este trabalho utilizou quatro etapas da Sequência de Ensino Investigativo, conforme está apresentado no Quadro 1.

Quadro 1: Etapas da SEI

\begin{tabular}{|c|l|l|}
\hline \multicolumn{1}{|c|}{} & \multicolumn{1}{|c|}{ Etapas } & \multicolumn{1}{|c|}{ Caracterização } \\
\hline 1 & $\begin{array}{l}\text { Apresentação e exposição } \\
\text { do conteúdo }\end{array}$ & $\begin{array}{l}\text { A exposição ocorreu, inicialmente, de forma teórica, } \\
\text { compreendendo definições e exemplos relativos ao } \\
\text { conteúdo de Le dos Senos e Lei dos Cossenos, } \\
\text { relacionando, de forma interdisciplinar com o estudo de } \\
\text { Vetores, estudo do campo da Física }\end{array}$ \\
\hline 2 & Experimentação teórica & $\begin{array}{l}\text { Neste momento foram apresentados e resolvidos } \\
\text { exemplos do conteúdo }\end{array}$ \\
\hline 3 & Experimentação prática & $\begin{array}{l}\text { Nesta etapa ocorreu a experimentação prática com a } \\
\text { utilização de exemplos que foram demonstrados no } \\
\text { simulador PhET }\end{array}$ \\
\hline 4 & Avaliação & $\begin{array}{l}\text { Os instrumentos avaliativos tomaram como base a } \\
\text { resolução de uma lista de exercícios, envolvendo o } \\
\text { conteúdo abordado }\end{array}$ \\
\hline
\end{tabular}

Fonte: Elaboração dos Autores (2020)

Etapa 1: Ocorreu a exposição do conteúdo relativo à Lei dos Senos e à Lei dos Cossenos, utilizando exemplos relacionados, de forma interdisciplinar, com o conteúdo de Vetores, geralmente, trabalhado em Física, sem se estabelecer correlações com a Matemática.

Etapa 2: durante a exploração do conteúdo, foram apresentados exemplos que permitiram, a partir da resolução, a exploração dos conteúdos propostos.

Etapa 3: Em seguida, aconteceu a demonstração do simulador que apresentou as Adições de Vetores: equações a partir da plataforma PhET. O objetivo foi demonstrar a 
adição e a subtração de vetores, relacionando-as com a Lei dos Senos e a Lei dos Cossenos. Nesta etapa, então, foram utilizados os mesmos exemplos teóricos trabalhados, de modo a permitir que fossem aplicados no simulador.

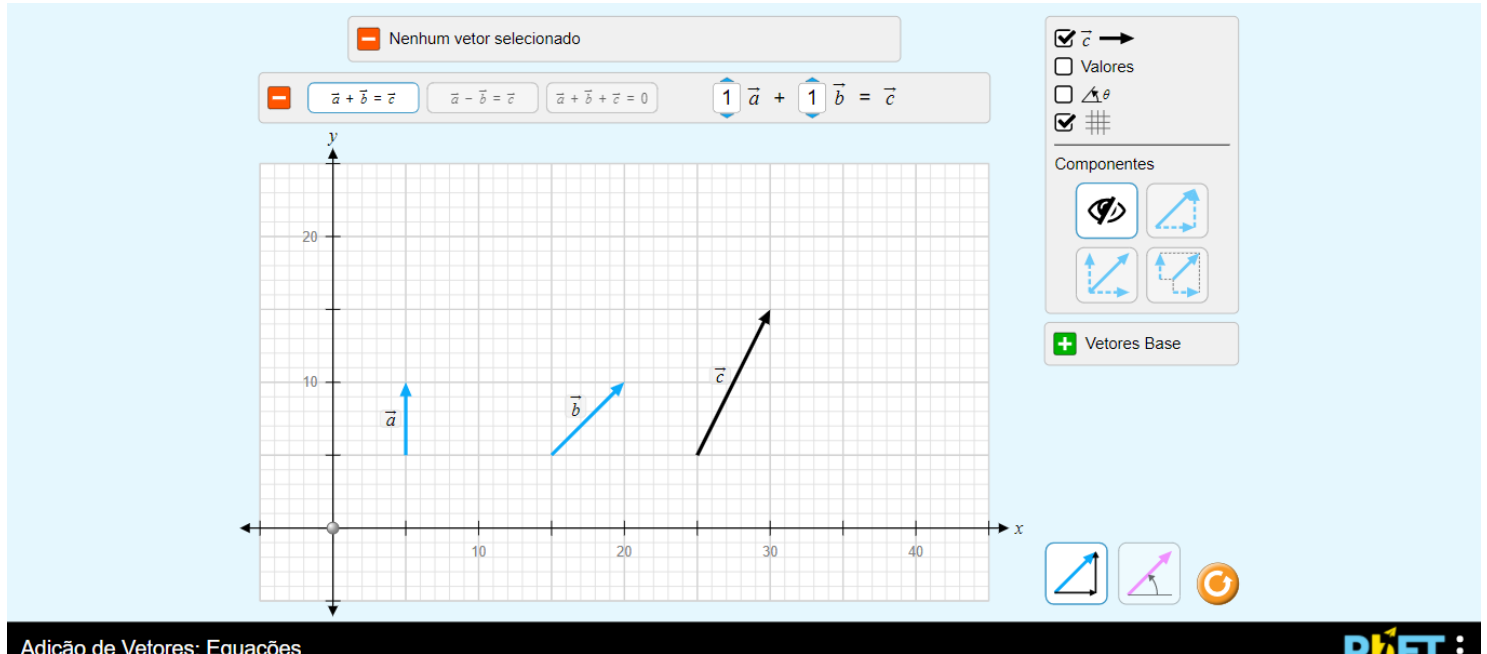

Figura 1: Interfase do simulador Adições de Vetores: equações da plataforma PhET (Acervo da Pesquisa)

Etapa 4: Os estudantes foram convidados a resolver quatro questões abertas sobre a Lei dos Senos e a Lei dos Cossenos, utilizando para isso as operações com Vetores. No Quadro 2 é possível visualizar as questões propostas como a atividade avaliativa.

Quadro 2: Atividade avaliativa

\begin{tabular}{|c|c|}
\hline Questões & Contextualização \\
\hline 1 & $\begin{array}{l}\text { Barbara quer fazer a moldura de um quadro para colocar sua pintura no } \\
\text { formato de um triângulo. Sabe-se que os vetores de comprimento medem } \\
\text { respectivamente } a_{x}=6 \mathrm{~cm} \text { e } a_{y}=4 \mathrm{~cm} \text {, formando entre si um ângulo de } \\
33,7^{\circ} \text {. Encontre a medida do terceiro lado que forma essa moldura. (Utilize } \\
\text { duas ordens decimais). }\end{array}$ \\
\hline 2 & $\begin{array}{l}\text { André decidiu fazer um origami com uma folha de papel, inicialmente } \\
\text { cortou-a no formato de um triângulo, tendo como vetores de comprimento } \\
a_{x}=7 \mathrm{~cm} \text { e } a_{y}=9 \mathrm{~cm} \text {, formando entre si um ângulo de } 52,1^{\circ} \text {. Calcule o } \\
\text { valor do terceiro lado desse origami. (Utilize duas ordens decimais). }\end{array}$ \\
\hline 3 & $\begin{array}{l}\text { Eduarda deseja cortar um tecido para colocar como tapete da sua casa, em } \\
\text { formato triangular. Sabe-se que a medidas dos vetores de comprimento } \\
\text { medem, respectivamente, } 4 \mathrm{~cm} \text { e } 7 \mathrm{~cm} \text {, formando um ângulo de } 60,3^{\circ} \text {. } \\
\text { Calcule a medida do terceiro lado desse triângulo. }\end{array}$ \\
\hline 4 & $\begin{array}{l}\text { João deseja fazer um jardim em sua casa no formato de um triângulo } \\
\text { sabendo que as medidas de seus vetores têm comprimentos iguais a } 6 \mathrm{~cm} \\
\text { e } 8 \mathrm{~cm} \text {, respectivamente e, formando entre eles um ângulo de } 53,1^{\circ} \text {. Calcule } \\
\text { o terceiro lado. }\end{array}$ \\
\hline
\end{tabular}

Fonte: Elaboração dos Autores (2020) 
Após isso, responderam a um questionário que continha cinco questões objetivas, a fim de que fosse verificado o resultado, ou seja, a eficácia ou não, de se trabalhar um mesmo conteúdo de modo interdisciplinar e ainda, com auxílio da Tecnologia. Essa então foi uma das ferramentas que permitiu chegar a um resultado da pesquisa-ação.

\section{Resultados e discussões}

Os resultados dessa pesquisa-ação são apresentados a seguir de duas formas: por meio de gráficos que mostram os índices de erros e de acertos dos estudantes por meio da atividade avaliativa e, em seguida, num único gráfico que apresenta o questionário de autoavaliação dos estudantes em relação aos processos de ensino e de aprendizagem propostos.

A primeira questão da atividade avaliativa consiste em um item que apresenta essa contextualização: "Bárbara quer fazer a moldura de um quadro para colocar sua pintura no formato de um triângulo. Sabe-se que os vetores de comprimento medem respectivamente $a_{x}=6 \mathrm{~cm}$ e $a_{y}=4 \mathrm{~cm}$, formando entre si um ângulo de $33,7^{\circ}$. Encontre a medida do terceiro lado que forma essa moldura (Utilize duas ordens decimais)”. Assim, na resolução, por meio do cálculo utilizando a Lei dos Cossenos, teve como objetivo encontrar o valor da medida do terceiro lado igual a, aproximadamente, $7,17 \mathrm{~cm}$. Diante do que é exposto na Figura 2, cerca de 66,6\% dos estudantes acertaram o item, apresentando o cálculo de forma satisfatória.

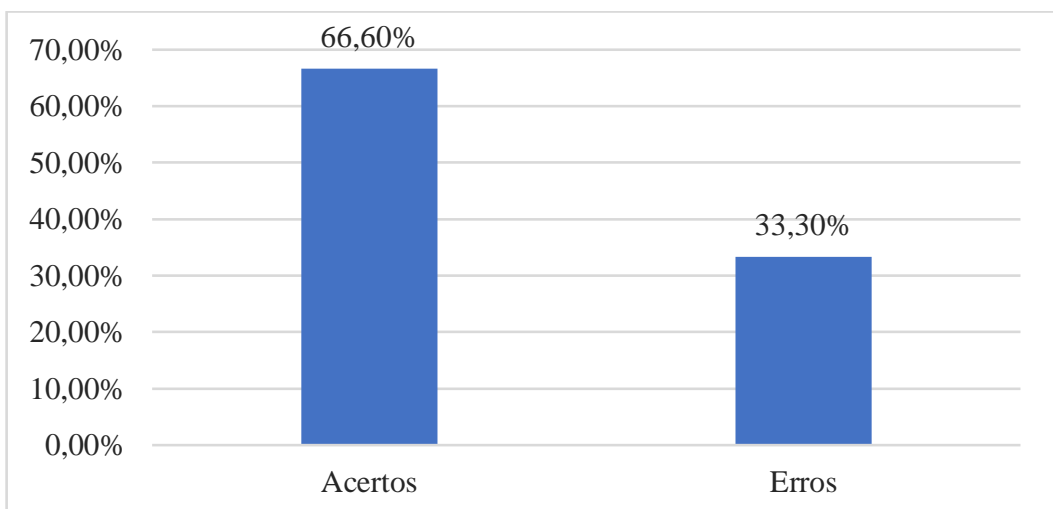

Figura 2: Primeira questão da atividade avaliativa (Elaboração dos Autores, 2020)

A segunda questão consistiu na seguinte resolução: “André decidiu fazer um origami com uma folha de papel, inicialmente cortou-a no formato de um triângulo, tendo como vetores de comprimento $a_{x}=7 \mathrm{~cm}$ e $a_{y}=9 \mathrm{~cm}$, formando entre si um ângulo de 
$52,1^{\circ}$. Calcule o valor do terceiro lado desse origami (Utilize duas ordens decimais)". Assim, ao desenvolver o cálculo utilizando a Lei dos Cossenos, o resultado obtido resultou em, aproximadamente, 11,35 cm. Na Figura 3, a seguir, é possível visualizar que $60 \%$ dos pesquisados apresentaram resultado satisfatório ao item.

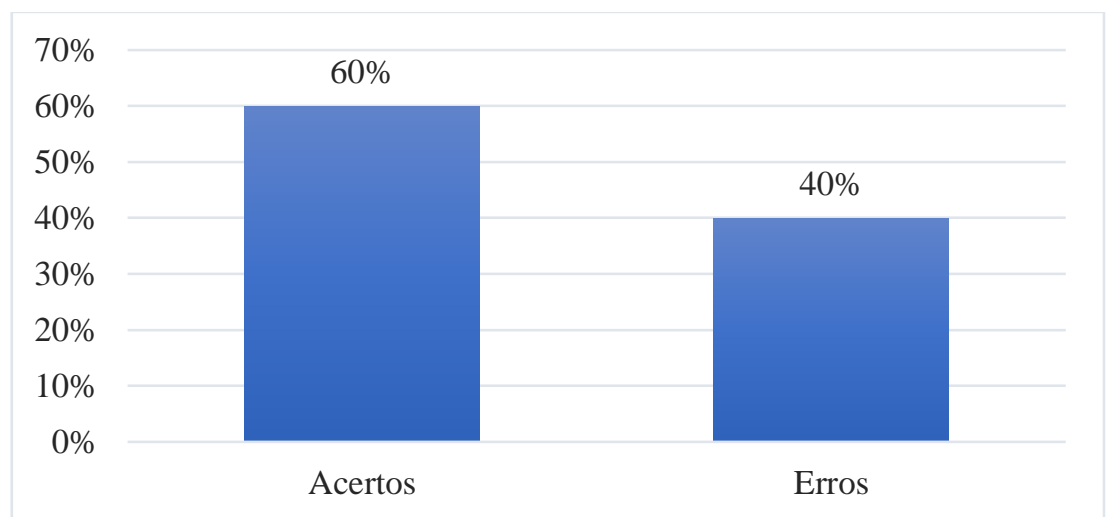

Figura 3: Segunda questão da atividade avaliativa (Elaboração dos Autores, 2020)

A terceira questão da atividade avaliativa consistiu em um item que apresentou essa contextualização: "Eduarda deseja cortar um tecido para colocar como tapete da sua casa, em formato triangular. Sabe-se que a medidas dos vetores de comprimento medem, respectivamente, $4 \mathrm{~cm}$ e $7 \mathrm{~cm}$, formando entre eles, um ângulo de $60,3^{\circ}$. Calcule a medida do terceiro lado desse triângulo". Assim, em um cálculo utilizando a Lei dos Senos, o resultado obtido foi, aproximadamente, $8,2 \mathrm{~cm}$. A Figura 4 mostra que $80 \%$ dos estudantes obtiveram resultado satisfatório à questão proposta.

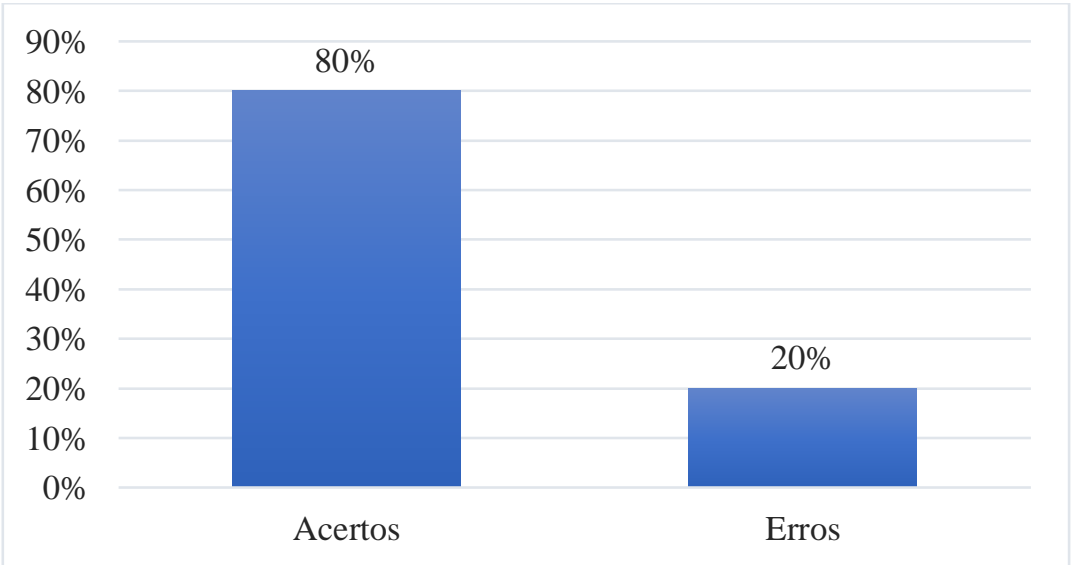

Figura 4: Terceira questão da atividade avaliativa (Elaboração dos Autores, 2020)

A questão quatro da atividade avaliativa foi assim contextualizada: "João deseja fazer um jardim em sua casa no formato de um triângulo, contendo as medidas de seus vetores de comprimentos iguais a $6 \mathrm{~cm}$ e $8 \mathrm{~cm}$, respectivamente, formando entre eles um ângulo de 53,1 $1^{\circ}$. Calcule a medida do terceiro lado". Assim, a resolução propiciou um 
cálculo utilizando a Lei dos Senos, de forma que o resultado fosse $10,2 \mathrm{~cm}$ para o terceiro lado do triângulo. A Figura 5 mostra que $80 \%$ dos estudantes acertaram o item.

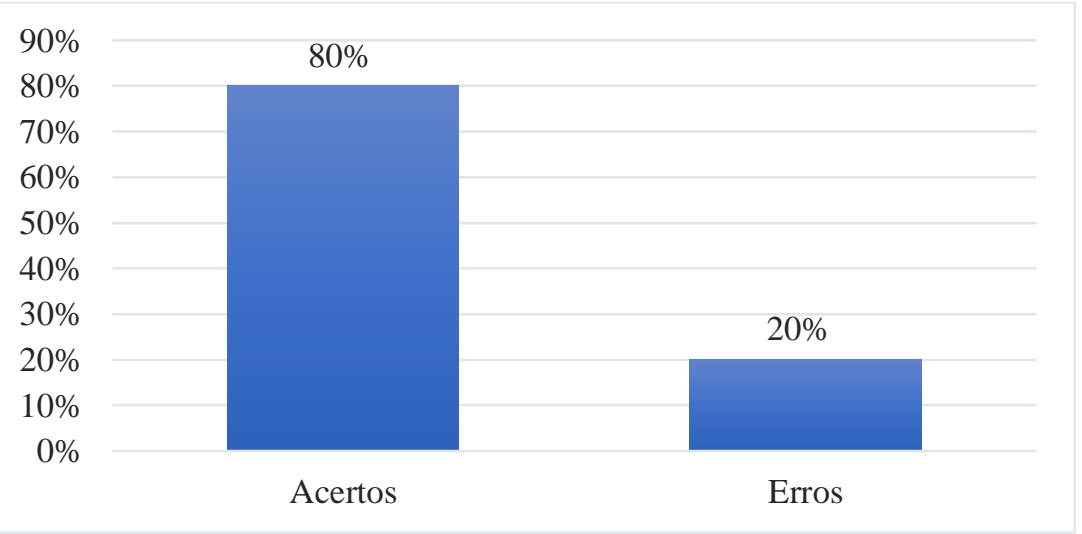

Figura 5: Quarta questão da atividade avaliativa (Elaboração do Autores, 2020)

Dessa forma, é possível apreender do que foi exposto, que houve uma interação dos estudantes com o conteúdo à medida que o objeto de aprendizagem era aplicado e, assim, favoreceu o ensino e a aprendizagem. Como admite Perius (2012), a Matemática precisa ser tanto vista como trabalhada, com uma maneira de pensar, como um processo em permanente evolução - não sendo apenas apresentada como algo pronto e acabado - mas possibilitando, dinamicamente, por parte do estudante, a construção e apropriação do conhecimento e de habilidades de raciocínio.

Moura, Ramos e Lavor (2020) ao utilizarem o simulador PhET no ensino de Trigonometria de forma interdisciplinar com o contexto problematizador que envolve o lançamento de projéteis, afirmam que os dados refletiram a compreensão do conteúdo exposto, pois conseguiram relacioná-los ao aplicar no simulador. Isso evidencia que o conhecimento, quando alia teoria e prática, é facilitado pela compreensão do conceitos. Neste exemplo, a resolução escrita é realizada por meio da equação de lançamento de projéteis e a aplicação dessas informações no simulador. Com isso, os discentes conseguem retirar informações das questões contextualizadas e transformá-las em dados. Isso torna a aula interativa e interdisciplinar, sendo perceptível a motivação e as trocas entre os estudantes.

Dessa forma, é compreensível o porquê que mediante a utilização dessa metodologia, utilizando o simulador PhET de forma interdisciplinar, os processos de ensino e de aprendizagem possibilita a interação e a motivação dos estudantes, tornando também a aula mais dinâmica. 
Os estudantes, ao serem convidados a responder o questionário de autoavaliação sobre a utilização do simulador PhET e suas contribuições para os processos de ensino e de aprendizagem, mostraram 90\% de satisfação pelo desempenho. Ao serem indagados a respeito da importância em utilizar as Tecnologias como recurso que contribuíssem para o ensino e a aprendizagem, $100 \%$ deles responderam que contribui de forma positiva. Sobre as dificuldade que os discentes sentiram em entender o simulador, $70 \%$ afirmaram não sentir dificuldades. $\mathrm{Na}$ questão que indagou se o simulador PhET ajudou na compreensão do conteúdo de Vetores com relação à Lei dos Senos e a Lei dos Cossenos, $90 \%$ dos estudantes afirmaram que isso ocorreu de forma satisfatória.

Por fim, no questionário de autoavaliação, 90\% dos estudantes responderam que ocorreu interatividade em todo processo de construção dos conteúdos propostos, tanto entre eles, quanto entre eles e pesquisadores.

Desse modo, é possível observar, por meio desses resultados apresentados, que a interdisciplinaridade entre Matemática e Física se torna mais favorecida, principalmente, quando se utiliza o simulador $\mathrm{PhET}$, como um importante recurso didático-metodológico e como instrumento de interação durante uma aula

\section{Considerações finais}

Este artigo apresentou uma Sequência de Ensino Investigativo (SEI) no estudo interdisciplinar de Trigonometria com a Lei dos Senos e a Lei dos Cossenos, utilizando o auxílio do simulador PhET como objeto de aprendizagem. Destacam-se, por meio dele, resultados positivos no requisito de aplicação, contribuição para o ensino e a aprendizagem dos conteúdos e interação efetiva nas relações estudante-estudante e estudante-pesquisador durante a aula.

Ao se visualizar que as aplicações dos objetos de aprendizagem, de forma interdisciplinar, o propósito foi oferecer uma melhor compreensão do conteúdo, de modo a propiciar aplicações concretas e visualmente possíveis de serem demonstradas, levando o estudante a entender as definiçõos e permitindo sua participação ativa no processo de aprendizagem. Desse modo, com a mediação da tecnologia, atuou como o protagonista de todo processo de construção dos conceitos propostos.

Espera-se que este trabalho possa reforçar a vertente da possibilidade de uma 
aprendizagem interdisciplinar, no que tange aos conteúdos de Trigonometria e Vetores, mediada por recursos tecnológicos adequados, de uma forma dinâmica, simples prática.

\section{Referências}

AFONSO, Tarley; MALDONADO, Michelli. Um exemplo prático do uso da tecnologia como meio para o ensino de Matemática. Proceeding Series Of The Brazilian Society Of Computational And Applied Mathematics, Uberlândia, v. 7, n. 1, p. 1-2, 2020.

AGUIAR, Eliane Vigneron Barreto; FLÔRES, Maria Lucia Pozzatti. Objetos de aprendizagem: conceitos básicos. In: TAROUCO, Liane Margarida Rockenbach; COSTA, Valéria Machado da; AVILA, Bárbara Gorziza; NEZ, Marta Rosecler; SANTOS, Edson Felix dos. (Org). Objetos de aprendizagem: teoria e prática. Porto Alegre: Evangraf, 2014, p. 12-28.

ARAUJO, Rafael Enrique Gutiérrez; BRACH, Luis Andrés Castillo. Simuladores com o software GeoGebra como objetos de aprendizagem para o ensino da Física. Tecné, Episteme y Didaxis, Bogotá, n. 47, p. 201-216, jan./jun. 2020.

BORGES, Leila Bernardes. Modelagem Matemática no ensino de Trigonometria. 2020. 156f. Dissertação (Mestrado Profissional em Matemática em Rede Nacional) — Instituto de Matemática e Estatística. Universidade Federal de Goiás. Goiânia.

BUENO, Simone; ALENCAR, Edvonete Souza de; MILLONES, Teresa Sofía Oviedo. Reflexões e desafios da resolução de problemas nas aulas de Matemática: um ensaio teórico. Educação Matemática Debate, Montes Claros, v. 1, n. 1, p. 9-27, jan. 2017.

CARVALHO, Ana Maria Pessoa de. O ensino de Ciências e a proposição de sequências de ensino investigativas. In: CARVALHO, Ana Maria Pessoa de. (Org.). Ensino de Ciências por investigação: condições para implementação em sala de aula. São Paulo: CENCAGE Learning, 2013, p. 1-20.

CRUZ, João Roberto Ursino da; MONTEIRO, Ana Maria. Requisitos para o desenvolvimento de objetos de aprendizagem reutilizáveis para dispositivos móveis. CONGRESSO BRASILEIRO DE INFORMÁTICA NA EDUCAÇÃO, 8, 2019, Brasília. Anais do CBIE2019. Brasília: SBC, 2019, p. 81-88.

FERNANDA, Edberto; REIS, Marcia Cristina dos. A semiótica e o design da informação no desenvolvimento de objetos de aprendizagem. Ciência da Informação, Brasília, v. 48, n. 1, p. 23-40, 2019.

LAVOR, Otávio Paulino; MARTINS, Kaliane Morais de Lucena. Potencializando o ensino de Matemática através de objetos de aprendizagem. E-Mosaicos, Rio de Janeiro, v. 9 , n. 21, p. 173-186, maio/ago. 2020.

MACHADO, Maxiel de Mesquita. GeoGebra: uma proposta para o ensino de funções trigonométricas. 2020. 184f. Dissertação (Mestrado Profissional em Matemática em Rede 
Nacional) — Unidade Acadêmica de Matemática e Tecnologia. Universidade Federal de Goiás. Catalão.

MASOLA, Wilson de Jesus; ALLEVATO, Norma Suely Gomes. Dificuldades de aprendizagem matemática: algumas reflexões. Educação Matemática Debate, Montes Claros, v. 3, n. 7, p. 52-67, jan. 2019.

MELO, Ângelo Gomes de; SANTO, Mirley Luciene dos; ARAÚJO, Cleide Sandra Tavares. A experimentação, a problematização e o uso de recursos digitais na aplicação de uma sequência didática para o ensino de soluções no Ensino Médio. Research, Society and Development, Vargem Grande Paulista, v. 9, n. 7, p. 1-30, jan. 2020.

MOURA, Patrícia de Souza; RAMOS, Maria do Socorro Ferreira; LAVOR, Otávio Paulino. Investigando o ensino de trigonometria através da interdisciplinaridade com um simulador da plataforma PhET. Reamec, Cuiabá, v. 8, n. 3, p. 573-591, set./dez. 2020.

OLIVEIRA, Gisele Pereira. O uso da história da Matemática e dos objetos de aprendizagem como ferramentas pedagógicas na formação de professores de Matemática. Boletim Cearense de Educação e História da Matemática, Fortaleza, v. 7, n. 20, p. 126-138, jan. 2020.

OLIVEIRA, Kaio Eduardo de Jesus; PORTO, Cristiane de Magalhães; ALVES, André Luiz. Memes de redes sociais digitais enquanto objetos de aprendizagem na Cibercultura: da viralização à Educação. Acta Scientiarum, Maringá, v. 41, p. 1-11, jan. 2019.

OLIVEIRA, Sandra Alves de; REZENDE, Dayselane Pimenta Lopes; REIS, Andreia Rezende Garcia; CARNEIRO, Reginaldo Fernando. Vivências de professoras dos Anos Iniciais no trabalho com a resolução de problemas em uma formação continuada. Educação Matemática Debate, Montes Claros, v. 5, n. 11, p. 1-27, jan. 2021.

PERIUS, Ana Amélia Butzen. A tecnologia aliada ao ensino de Matemática. 2012. 49f. Monografia (Especialização em Mídias na Educação) - Centro Interdisciplinar de Novas Tecnologias na Educação. Universidade Federal do Rio Grande do Sul. Cerro Largo.

RODRIGUES, Macelo Melazzo. Modelagem Matemática da voz, Trigonometria e Robótica: atividades interativas. 2020. 193f. Dissertação (Mestrado em Ensino de Ciências e Matemática) — Faculdade de Matemática. Universidade Federal de Uberlândia. Uberlândia.

SANTOS, Fernanda dos. Argumentação em uma sequência de ensino investigativa envolvendo Química Forense. 2020. 139f. Dissertação (Mestrado em Ensino de Ciências e Matemática) - Centro de Ciências Exatas e Tecnologia. Universidade Federal de Sergipe. São Cristóvão.

SILVA, Carla Martins da; TEIXEIRA, Eliana Maria Mallmann; MÜLLER, Thaísa Jacintho. Objetos de aprendizagem no ensino de Matemática: uma possibilidade no ensino e aprendizagem das frações. Revista Sergipana de Matemática e Educação Matemática, Itabaiana, v. 4, n. 1, p. 82-96, jan./jun. 2019. 
SILVA, Dalmo Rodrigues da; SEVERIANO, Eusiléa Pimenta Roquete; URZÊDA, Rhêmora Ferreira da Silva. A utilização de AVA's, apps e games na aprendizagem de Matemática em um curso de Pedagogia. Revista de Ciências Sociais Aplicada, Santos, v. 1, p. 1-5, jan. 2020.

TADIELLO, Rafaela Bressan. Sequência de ensino investigativa e práticas laboratoriais: novos olhares sobre o ensino de Ciências. 2020. 169f. Dissertação (Mestrado em Educação em Ciências: Química da Vida e Saúde) — Instituto de Ciências Básicas da Saúde. Universidade Federal do Rio Grande do Sul. Porto Alegre. 